\title{
Why soft X-ray transients can remain in the low/hard state during outburst
}

\author{
E. Meyer-Hofmeister
}

\author{
Max-Planck-Institut für Astrophysik, Karl- Schwarzschildstr. 1, 85740 Garching, Germany \\ e-mail: emm@mpa-garching.mpg.de
}

Received 2 March 2004 / Accepted 3 May 2004

\begin{abstract}
In the canonical understanding of transient X-ray sources the accretion during quiescence occurs via a geometrically thin disk in the outer part and via an advection-dominated hot coronal flow/ADAF in the inner part. The inner part important for the radiation yields a hard spectrum. In most sources the luminosity increase during outburst causes a change over to a soft spectrum which can be understood as that of a multi-color black body disk reaching inward to the marginally stable orbit. A few transient sources do not display this transition to the soft state. We show that this can be understood as due to relatively low peak luminosities in outburst in systems with short orbital periods and therefore less mass accumulated in the then smaller accretion disk. This is in agreement with the observations which show that most likely short orbital period systems remain in the hard spectral state.
\end{abstract}

Key words. accretion, accretion disks - black hole physics - X-rays: binaries - stars: individual: XTE J1118+480 stars: individual: GRO J0422

\section{Introduction}

Accretion onto stellar-mass black holes in binaries is of interest in connection also with supermassive black holes with relevance to galaxy formation and cosmological questions. Since the first identification of a black hole X-ray binary, Cygnus X-1, by Webster \& Murdin (1972) and Bolton (1972), 30 years ago, several black hole X-ray binaries, persistently bright or transient were found. According to the nature of the secondary star systems with a high-mass companion, usually a massive $\mathrm{O} / \mathrm{B}$ star, are classified as high-mass X-ray binaries (HMXB) and systems with a low-mass companion, of a few $M_{\odot}$ or less, as low-mass X-ray binaries (LMXB). 18 confirmed black hole binaries are now identified. A description of their properties and a discussion of the relevant issues is given in the recent review by McClintock \& Remillard (2003), a description of the observations in different wavelength regions can be found in the review by Charles \& Coe (2003). The gas flowing towards the black hole originates either from a wind of the high-mass companion (in HMXBs) or from Roche lobe overflow (in LMXBs). In both cases an accretion disk forms around the compact star.

Well known are the wind accreting systems with black holes of $\geq 10 M_{\odot}$ and high accretion rates, accordingly a fully ionized accretion disk and appearing as persistently bright $\mathrm{X}$-ray sources. Based on a recent high-resolution survey carried out with Chandra, Wang et al. (2002) report on faint sources which might also be wind accreting sources, but mainly neutron star wind accretors (Pfahl et al. 2002). Mass overflow rates from the low-mass companion stars are usually lower so that the gas is accumulated in a cool quiescent disk until an outburst is triggered. These outburst cycles caused by a disk instability are a feature which LMXBs have in common with dwarf novae. In several LMXBs the outburst recurrence time is so long that only one outburst was observed since the beginning of X-ray observations. The systems only detected during such an outburst were called X-ray novae. During quiescence the accretion rate is low, an advection-dominated accretion region fills the inner part, and the spectrum is hard. Usually in outburst the spectrum changes to a soft multi-color black body spectrum, which is interpreted as the radiation from a geometrically thin disk reaching inward to the innermost stable orbit. Such changes from a low/hard to a high/soft state and back are observed for several black hole LMXB outbursts and also in persistently bright HMXBs as well as in neutron stars.

Surprisingly a few LMXBs remain in the low/hard state during outburst, best observed for XTE J1118+480 and GRO J0422+32. It turns out that whether a transition to the high/soft state occurs or not depends on the peak mass flow rate in outburst. Only if this mass flow is high enough to overcome evaporation the disk can reach to the innermost stable orbit and the spectrum can become soft. The process of evaporation of matter from the disk into a corona therefore is a key linebreak feature for the understanding of the spectral transitions. The maximal evaporation rate, critical for spectral state transition, was determined from the coronal evaporation model (Meyer et al. 2000a) Thus for low peak luminosity the X-ray binary sources might remain in the low/hard state. The aim of the 
present investigation is to inquire about this special case studying the matter accumulation in the thin disk in dependence on the parameters disk size (orbital period), black hole mass, outburst recurrence time and the resulting peak luminosity. It is an interesting check of the evaporation model to compare the luminosity at which the state transition is observed (Maccarone 2003) with the theoretical prediction.

In Sect. 2 we briefly review the basic modes of accretion and the change over from disk accretion to an ADAF. In Sect. 3 we describe the dependence of the accumulation of gas in the accretion disk during quiescence on the system parameters. The resulting expected peak luminosity reached in outburst and the consequences for the spectral transition are discussed in Sect. 4. In Sect. 5 we compare with observations. Discussion and conclusions follow.

\section{Accretion disks in $\mathrm{X}$-ray binaries}

\subsection{Disk instability model}

As found in connection with the understanding of the cause of dwarf nova outbursts (Osaki 1974; Meyer \& Meyer-Hofmeister 1981; Smak 1984), for a certain range of surface density, the disk can be either in a cool state, where hydrogen and helium are unionized and the mass flow rate is low or in a hot state where the gas is ionized and the mass flow rate is high. In the cool state mass is accumulated until a surface density is reached where only the hot state is possible. The visual luminosity can then increase by more than a factor 100 . It is generally accepted that the outbursts of X-ray novae are caused by the same mechanism.

But some additional features are important. The orbital periods of black hole X-ray binaries are longer than those of dwarf novae because the companion stars have larger Roche lobes due to higher mass. Irradiation affects the disk in X-ray binary outbursts (Mineshige \& Kusunose 1993) and also can lead to accretion of most of the disk matter onto the black hole in the late outburst (King \& Ritter 1998). An even more important feature for the disk evolution in quiescence and the outburst is the evaporation of the inner disk, that causes the change over from accretion via a thin disk to a hot coronal flow (Meyer et al. 2000b). Due to this evaporation process a continuous mass flow towards the black hole prolongs the time needed for the accumulation of matter to a critical surface density. For a system like A0620-00 only $\frac{1}{3}$ of the gas transferred from the companion star is accumulated in the disk (Meyer-Hofmeister \& Meyer 1999). This explains the very long outburst repetition time. Since the evaporation efficiency increases strongly with the mass of the compact star evaporation is much more important in accretion disks around black holes than in dwarf nova accretion disks around white dwarfs (Meyer-Hofmeister 1998; Mineshige et al. 1998).

\subsection{Critical accretion rate for the spectral state transition}

The rate at which gas evaporates from the thin cool disk into the corona increases with decreasing distance from the black
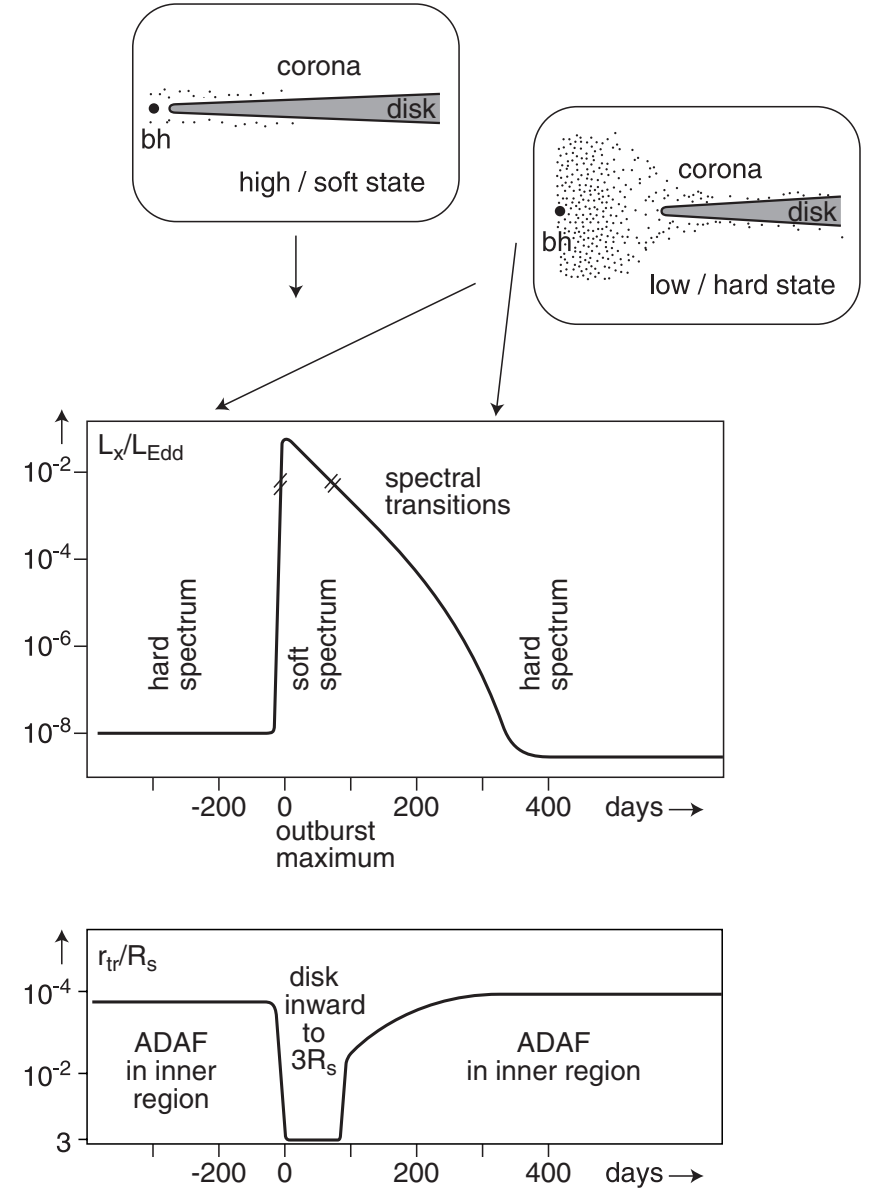

Fig. 1. Schematic description of an X-ray nova outburst: X-ray luminosity $L_{\mathrm{X}}$ measured in Eddington luminosity $L_{\mathrm{Edd}}$, transition radius $r_{\text {tr }}$ from a thin disk to a pure coronal flow measured in Schwarzschild radius $R_{\mathrm{S}}$, and related spectral states (numbers appropriate for A0620-00).

hole. An inner edge of the disk results where all the mass flow is transferred to the corona, inside accretion then occurs via a pure coronal flow/ADAF. Thus the evaporation efficiency (Meyer et al. 2000b) determines a disk truncation. This efficiency however reaches a maximum at a distance of about 300 Schwarzschild radii. The consequence is that for mass flow rates higher than this maximum the disk cannot be truncated anymore, instead continues inward to the last stable orbit. The critical mass flow rate corresponds to $0.02 L_{\mathrm{x}} / L_{\mathrm{Edd}}$ $\left(L_{\text {Edd }}=1.3 \times 10^{38}\left(M / M_{\odot}\right) \mathrm{erg} \mathrm{s}^{-1}\right)$. Taking into account some uncertainty in Fig. 1 we mark a range of 0.02 to $0.05 L_{\mathrm{x}} / L_{\mathrm{Edd}}$ (gray area).

\subsection{Advection-dominated accretion}

The X-ray flux observed from the X-ray nova A0620-00 in quiescence is very low compared to the optical flux from the outer disk regions (McClintock et al. 1995). This low flux can be explained if most of the thermal and kinetic energy is carried into the black hole by an advection-dominated accretion flow (ADAF) and is not radiated away. The successful description of the observed spectra (Narayan et al. 1997, 1999) confirmed 
this picture. Generally the two-temperature ADAF model provides a natural explanation for the low luminosities (see review Narayan et al. 2002). The coronal gas in the inner disk region emits a hard spectrum. In outburst when the mass flow rate increases above the critical rate the ADAF region disappears and the spectrum changes to a soft multi-color black body spectrum (Esin et al. 1998). These changes are observed for several LMXB outbursts and also in persistently bright HMXBs. Esin et al. (1997) described the configuration of the accretion flow in different states as the very high, high, intermediate, low and quiescent state. Our investigation concerns the high and low state. The quiescent state is not distinct from the low state, is just a version of extremely low mass flow (Narayan et al. 2002).

Figure 1 gives a schematic description of the changes of the luminosity, the location of the inner edge of the disk and the spectral states during an X-ray nova outburst.

In the following investigation we use "advection-dominated accretion flow" as the alternative to thin disk accretion, regardless of the specific forms and modifications suggested by various authors to meet the constraints from the observations in different wavelengths. Since the physics of the hot accretion flow seems to be very complex we limit our analysis to the investigation of the spectral state transition as triggered by the balance between evaporation efficiency and mass flow rate in the accretion disk.

\section{Accumulation of gas in the accretion disk during quiescence}

The amount of mass accumulated in the accretion disk during quiescence determines the peak mass flow rate in the outburst and therefore whether the hole caused by evaporation in the inner disk disappears.

In earlier work (Meyer-Hofmeister \& Meyer 1999, 2000) we investigated the mass accumulation in disks of classical $\mathrm{X}$-ray novae for a variety of system parameters, especially appropriate for modeling A0620-00.

The accumulation of gas in the disk depends on the mass overflow rate from the secondary star, on the disk size, and on the mass of the black hole. The black hole mass is an important parameter, a larger mass causes a more extended hole in the inner disk and the critical surface density can only be reached at a radius larger than that in the case of a lower mass primary with less evaporation. The amount of mass accumulated increases with disk size, i.e. orbital period. The mass overflow rate has a small effect only. A higher rate leads to an earlier outburst. The range of values we show corresponds to a recurrence time $\geq 10$ years. In Fig. 2 we show the amount of gas accumulated at outburst onset as a function of the orbital period for two black hole masses. The values depend on the frictional parameter $\alpha$. We modeled A0620-00 using the value $\alpha=0.05$. We were able to find agreement in several aspects: (1) the amount of mass accumulated in agreement with the amount deduced from the outburst lightcurve; (2) the low mass flow rate in the disk in agreement with the predictions from the ADAF based spectral fit; (3) agreement between theoretical and observed recurrence time (58 years assuming no outburst was missed in between). This result assumed a $4 M_{\odot}$

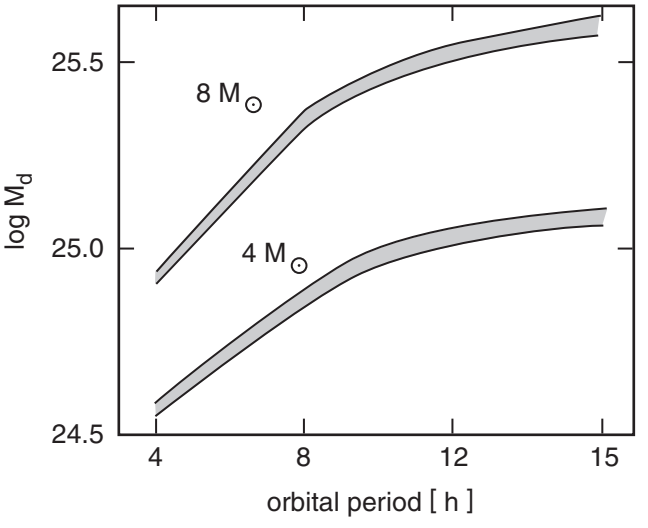

Fig. 2. Mass $M_{\mathrm{d}}[\mathrm{g}]$ accumulated in the accretion disk at the onset of the X-ray nova outburst. The range of values for given black hole mass and orbital period results from different mass overflow rates from the companion star (corresponding to different recurrence times. We consider the situation for matter accumulation in disks for recurrence times $\geq 10$ years) (from Meyer-Hofmeister \& Meyer 2000, Fig. 3).

black hole, the favored value at this time. Meanwhile it became clear that the black hole mass in A0620-00 is higher and that the distance is smaller. (To get good agreement with the observations a slightly higher viscosity would then be appropriate. Estimates can be deduced from the earlier computations. In Fig. 3 to account for such uncertainty we also add peak luminosities for half the amount of matter accumulated evaluated before.)

\section{Theoretically determined peak luminosity in outburst}

We use the results for disk evolution during quiescence to evaluate the maximal mass flow rate in the hot disk in outburst. We assume that with the onset of the outburst the disk immediately assumes the structure of the quasi-stationary accretion flow. This is an approximation to the real situation where this process takes a short time during which a small amount of gas already is accreted before the outburst maximum is reached.

The mass flow rate in the hot disk was calculated according to the standard relation between mass flow rate, mass in the disk, primary mass and disk size for a viscosity parameter $\alpha=0.2$ (Frank et al. 1985). As expected, the mass flow rate is higher for disks with longer orbital period. But for even larger disks (compare Fig. 3) the rates tend to level off due to the then longer diffusion time in the disk. For the correspondence between luminosity and mass accretion rate we used the formula $L=0.1 \dot{M} c^{2}$ ergs/s. This relation is appropriate for the soft state and should hold for the transition. For the hard state the luminosity might be somewhat smaller which could appear as lower values in the observations. The derived peak luminosities have to be compared with the critical value for the spectral transition. Though as noted above our procedure probably overestimates the absolute values of the peak luminosities the curves in Fig. 3 can be used as an illustration of the trend: low peak luminosities for systems with short orbital periods. Therefore one would expect that those systems that remain in the hard state would generally have short orbital periods. 


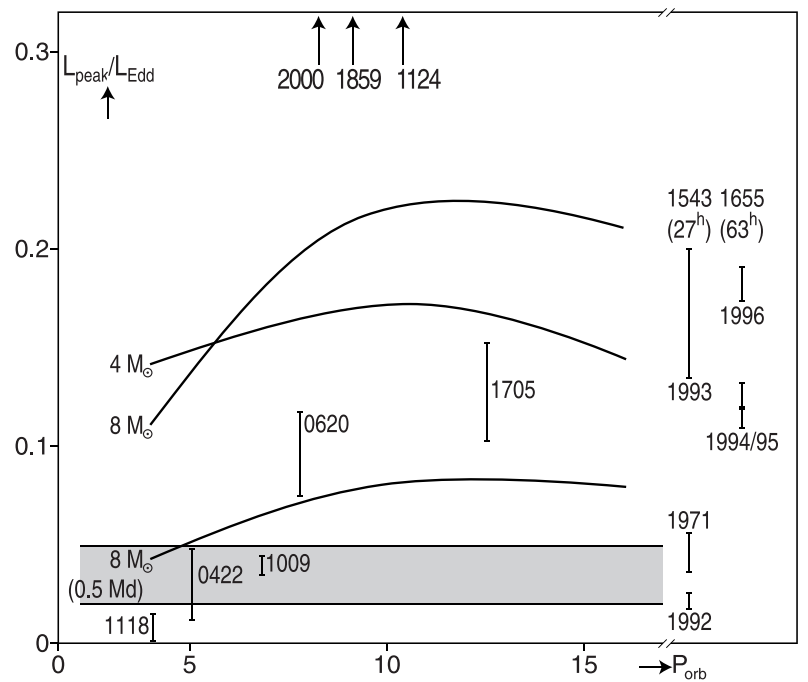

Fig. 3. Solid lines and dashed line: theoretically determined peak luminosities in outburst as function of orbital period; gray area: range for critical luminosity for spectral state transition; circles: observational results for black hole $\mathrm{X}$-ray transients, values larger than 0.3 are indicated by arrows in the uppermost part of the figure.

\section{Comparison with observations}

We now test whether the observed peak luminosities of systems which show a hard to soft state transition lie above the critical luminosity and those of systems which remain in the hard state in outburst lie below the critical luminosity. This can only be performed for systems where black hole mass and distance are known. This reduces the comparison to a small number of binaries (compare also Maccarone 2003). We list the data used here in Table 1.

\subsection{Observed peak luminosities in outburst}

We take the peak luminosities given by Chen et al. (1997) available at that time, for XTE J1118+480 the value from Remillard et al. (2000) and for XTE J1859+226 the value from Markwardt (2001). We did not include the data for the 1983 outburst of 4U1543-47 because of the only very short time observation. During the last years improved estimates for black hole masses and distances have been obtained for several systems, e.g. for A620-00 the black hole mass accepted earlier was 4.9-10 $M_{\odot}$ (Chen et al. 1997), while now McClintock \& Remillard (2003) obtain 8.7-12.9 $M_{\odot}$ and the distance estimate changed from 0.87 to $1.2 \mathrm{kpc}$. New optical and infrared photometry of GRO J0422+32 (Gelino \& Harrison 2003) lead to a determination of the black hole mass of $3.97 \pm 0.95 M_{\odot}$ with an adopted distance of $2.49 \pm 0.30 \mathrm{kpc}$. We have not included in our analysis sources with orbital periods much longer than the range covered by our evolutionary results. Certainly e.g. GRS $1915+105$ would be an interesting example to study the spectral state transitions, but for the larger accretion disks additional investigations would be needed to understand the observations. In Table 1 we give for all sources considered here. the values of the peak luminosity in outburst according to the new distance estimates The uncertainty in distance (up to 25\%) causes an uncertainty in the peak luminosity. In the Eddington luminosity an even larger uncertainty arises from the uncertainty of the black hole mass (we used the arithmetic mean value). We plot the observed peak luminosities in Fig. 3 but the just mentioned uncertainties in $L_{\text {peak }} / L_{\text {Edd }}$ should be kept in mind.

The observed peak luminosities differ surprisingly from source to source. While some values lie in the same range as the theoretically predicted numbers the peak luminosity of the sources GS 2000-251, XTE J1859+226, GRS 1124-684 and H1705-25 is very high, up to significant fractions of the Eddington luminosity. From disk evolution for sources with similar disk size and black hole mass one would expect a similar amount of matter accumulated. As mentioned earlier a difference in the mass overflow rate from the secondary star has little influence as long as we consider transient sources with long recurrence time. In these systems a high mass transfer rate is excluded, it would lead to a short outburst recurrence time. Such a large amount of gas as is necessary for the observed high mass flow rate in outburst can only be accumulated in the disk if the viscosity is particularly low, possibly related to a low magnetic activity of the secondary star.

\subsection{Luminosities at spectral state transitions}

In our analysis we compare peak luminosity with the critical luminosity for state transition. Theory predicts this value to be about 0.02 in Eddington luminosity (Meyer et al. 2000a). This is strongly supported by the recent analysis of observational data by Maccarone (2003) who finds that the luminosities at soft to hard state transition of 4 transient black hole $\mathrm{X}$-ray sources, 2 persistent black hole binaries and 3 neutron stars all lie in the range of $1-4 \%$ of the Eddington luminosity. To observe the hard to soft transition, usually during the early outburst rise, is more difficult. The observations seem to indicate that the luminosity at which that transition occurs is higher than that of the transition back to the hard state. The existence of such a "hysteresis effect" was first pointed out by Miyamoto et al. (1995), indicated also by later observations for a number of systems. The difference in luminosity lies around a factor of five (for a recent discussion see e.g. Zdziarski et al. 2004). In our Fig. 3 we take a range for the critical luminosity. The "hysteresis effect" will be investigated in work in progress (Meyer-Hofmeister et al. 2004).

\subsection{Sources which remain in the low/hard state during the outburst}

Observations have shown that a few sources have spectra which do not change to the soft/high state during an outburst, but remain in the hard state. Brocksopp et al. (2001) list the following systems: GS 1354-64, GRO J0422+32, GRS 1719 (1716) and XTE J1118+480. GS 1354-64 had three (or four) outbursts during two of which the spectrum remained hard. It is worth noting that these two also had significantly lower count rates indicating significantly lower peak luminosities. Another source that remains in the hard state, GRS 1737-31, 
Table 1. Peak luminosities of X-ray transients established as black-hole sources.

\begin{tabular}{lllllllll}
\hline \hline $\begin{array}{l}\text { Source } \\
\text { name }\end{array}$ & $\begin{array}{l}\text { Alternative } \\
\text { name }\end{array}$ & BH mass & $\begin{array}{l}\text { Companion } \\
\text { star }\end{array}$ & $\begin{array}{l}\text { Orbital } \\
\text { period } \\
\left(M_{\odot}\right)\end{array}$ & $\begin{array}{l}\text { Outburst } \\
\text { year }\end{array}$ & Distance & $\log L_{\text {peak }}$ & $\begin{array}{c}L_{\text {peak }} \\
L_{\text {Edd }}\end{array}$ \\
\hline XTE J1118+480 & KV UMa & $6.5-7.2$ & K5/M0V & 4.1 & 2000 & $1.8 \pm 0.5$ & 35.60 & 0.0005 \\
GRO J0422+32 & V518 Per & $3.97 \pm 0.95$ & M1V & 5.1 & 1992 & $2.49 \pm 0.3$ & 37.40 & 0.049 \\
GRS1009-45 & MM Vel & $6.3-8.0$ & K7/M0V & 6.8 & 1993 & $5.0 \pm 1.3$ & 37.99 & 0.106 \\
A0620-00 & V616 Mon & $8.7-12.9$ & K4V & 7.8 & 1975 & $1.2 \pm 0.1$ & 38.37 & 0.169 \\
GS2000+251 & QZ Vul & $7.1-7.8$ & K3/K7V & 8.3 & 1988 & $2.7 \pm 0.7$ & 38.86 & 0.755 \\
XTE J1859+226 & V406 Vul & $7.6-12:$ & - & $9.2:$ & 1999 & 11 & 38.86 & 0.574 \\
GS1124-684 & GU Mus & $6.5-8.2$ & K3/K5V & 10.4 & 1991 & $5 \pm 1.3$ & 38.78 & 0.636 \\
H1705-25 & V2107 Oph & $5.6-8.3$ & K3/7V & 12.5 & 1977 & $8 \pm 2$ & 38.58 & 0.425 \\
4U1543-47 & IL Lupi & $7.4-11.4$ & A2V & 27.0 & 1971 & $7.5 \pm 0.5$ & 38.83 & 0.559 \\
& & & & & 1992 & & 38.48 & 0.249 \\
GRO J1655-40 & V1033 Sco & $6.0-6.6$ & F3/F5IV & 62.9 & 1994 & $3.2 \pm 0.2$ & 37.86 & 0.089 \\
& & & & & 1995 & & 37.77 & 0.073 \\
& & & & & 1996 & & 38.02 & 0.129 \\
\hline
\end{tabular}

Note: Black hole mass, spectral type of companion star, orbital period, outburst year and distance as listed in McClintock \& Remillard (2003). Peak luminosities in outburst $L_{\text {peak }}$ taken from Chen et al. (1997, Table 8), values here determined with respect to the more recent black hole mass and distance estimates given above; Values for XTE J1118+480 from Remillard et al. (2000), for GRO J0422+32 from Gelino \& Harrison (2003), for XTE J1859+226 from Markwardt (2001). Eddington luminosities according to the listed black hole masses.

was mentioned by McClintock \& Remillard (2003). In very recent work Brocksopp et al. (2004) list a few more sources as possible hard state candidates. Of the systems listed above only for GRO J0422+32 and XTE J1118+480 black hole masses are known. These systems are plotted in Fig. 3. Indeed the peak luminosities are very low and the orbital periods are short as expected.

\section{Conclusions}

The peak luminosities reached during outburst discriminate between systems in which, for high peak luminosity, a transition to the soft spectral state occurs, and, those in which, for low peak luminosity, the spectrum remains hard. For low peak luminosity, i.e. low mass flow rate in the disk, coronal evaporation truncates the thin disk even in outburst, further inward an advection-dominated accretion flow occurs and the spectrum stays hard. For high peak luminosity instead the disk cannot be truncated, the thin disk reaches inward to the last stable orbit and the spectrum is a soft multi-color black body spectrum. We have discussed the dependence on disk size and black hole mass. We expect the X-ray novae with the shortest orbital periods to eventually remain in the low/hard state throughout the outburst. This agrees with the observations for XTE J1118+480 and GRO J0422+32.

For a few sources, GS 2000+251, XTE J1859+226 and GRS 1124-684 the observed peak luminosity is very high. An earlier investigation (Meyer-Hofmeister \& Meyer 2000) had shown that, in systems with long recurrence time, the mass overflow rate from the secondary star only weakly influences the mass accumulated during quiescence. Therefore the large amount of matter accumulated in these disks cannot be due to an enhanced mass overflow rate. We argue a peak luminosity of a significant fraction of the Eddington luminosity must be related to a lower viscosity in the cool disk. Since the origin of the viscosity in the cool disk possibly lies in the magnetic fields reaching over from the companion star (Meyer \& Meyer-Hofmeister 1999) this leads to the question whether the magnetic fields of the secondary stars of these systems are less strong.

\section{References}

Bolton, C. T. 1972, Nature, 240, 124

Brocksopp, C., Jonker, P. G., Fender, R. P., et al. 2001, MNRAS, 323, 517

Brocksopp, C., Bandyopadhyay, R. M., \& Fender, R. P. 2004, New Astron., 9, 249

Charles, P. A., \& Coe, M. J. 2003, in Compact Stellar X-ray Sources, ed. W. H. G. Lewin, \& M. van der Klis [arXiv: astro-ph0308020]

Esin, A. A., McClintock, J. E., \& Narayan, R. 1997, ApJ, 489, 865

Esin, A. A., Narayan, R., Ciu, W., et al. 1998, ApJ, 505, 854

Frank, J., King, A. R., \& Raine, D. J. 1985, Accretion power in astrophysics (Cambridge University Press)

Gelino, D. M., \& Harrison, T. E. 2003, ApJ, 599

King, A., \& Ritter, H. 1998, MNRAS, 293, L42

Maccarone, T. J. 2003, A\&A, 409, 697

Markwardt, C. 2001, Ap\&SS, 276, 209

McClintock, J. E., \& Remillard, R. A. 2003, to appear in Compact Stellar X-ray Sources, ed. W. H. G. Lewin, \& M. van der Klis (Cambridge University Press) [arXiv: astro-ph 0306213]

McClintock, J. E., Horne, K., \& Remillard, R. A. 1995, ApJ, 513, 811 
Meyer, F., \& Meyer-Hofmeister, E. 1981, A\&A, 104, L10

Meyer, F., \& Meyer-Hofmeister, E. 1999, A\&A, 341, L23

Meyer, F., Liu, B. F., \& Meyer-Hofmeister, E. 2000a, A\&A, 354, L67

Meyer, F., Liu, B. F., \& Meyer-Hofmeister, E. 2000b, A\&A, 361, 175

Meyer-Hofmeister, E., \& Meyer, F. 1999, A\&A, 348, 154

Meyer-Hofmeister, E., \& Meyer, F. 2000, A\&A, 355, 1073

Meyer-Hofmeister, E., Meyer, F., \& Liu, B. F. 1998, A\&A, 339, 507

Mineshige, S., \& Kusunose, M. 1993, PASJ, 45, 113

Mineshige, S., Liu, B. F., Meyer, F., et al. 1998, PASP, 50, L5

Miyamoto, S., Kitamoto, S., Hayashida, K. S., et al. 1995, ApJ, 442, L13

Narayan, R. 2002, in Lighthouses of the Universe, Conf. Proc., ed. M. Gilfanov, R. Sunyaev, \& E. Churazov (Springer) ESO Astroph. Symp., 405
Narayan, R., McClintock, J. E., \& Yi, I. 1996, ApJ, 457, 821

Narayan, R., Barret, D., \& McClintock, J. E. 1997, ApJ, 482, 448

Narayan, R., Garcia, M. R., \& McClintock 2002, in Proc. IX Marcel Grossmann Meeting, ed. V. Gurzadyan, R. Jantzen, \& R. Ruffini (Singapore: World Scientific), Part A, 405

Osaki, Y. 1974, PASJ 26, 429

Pfahl, E., Rappaport, S., \& Podsiadlowski, P. 2002, ApJ, 571, L37

Remillard, R., Morgan, E., Smith, D., et al. 2000, IAU Circ., 7389

Smak, J. 1984, Acta Astron., 34, 161

Wang, Q. D., Gotthelf, E. V., \& Lang, C. C. 2002, Nature, 415, 148

Webster, B. L., \& Murdin, P. 1972, Nature, 235, 37

Zdziarski, A. A., Gierliński, M., Mikolajewska, J., et al. 2004 [arXiv: astro-ph/0402380] 